\title{
Percepción y uso del outsourcing de contabilidad de las MiPyMEs: evidencia empírica en Panamá
}

\author{
Carlos Antonio Changmarín Reyes \\ Universidad de Panamá
}

Recibido: 11 de septiembre de 2012

Aceptado: 3 de mayo de 2013

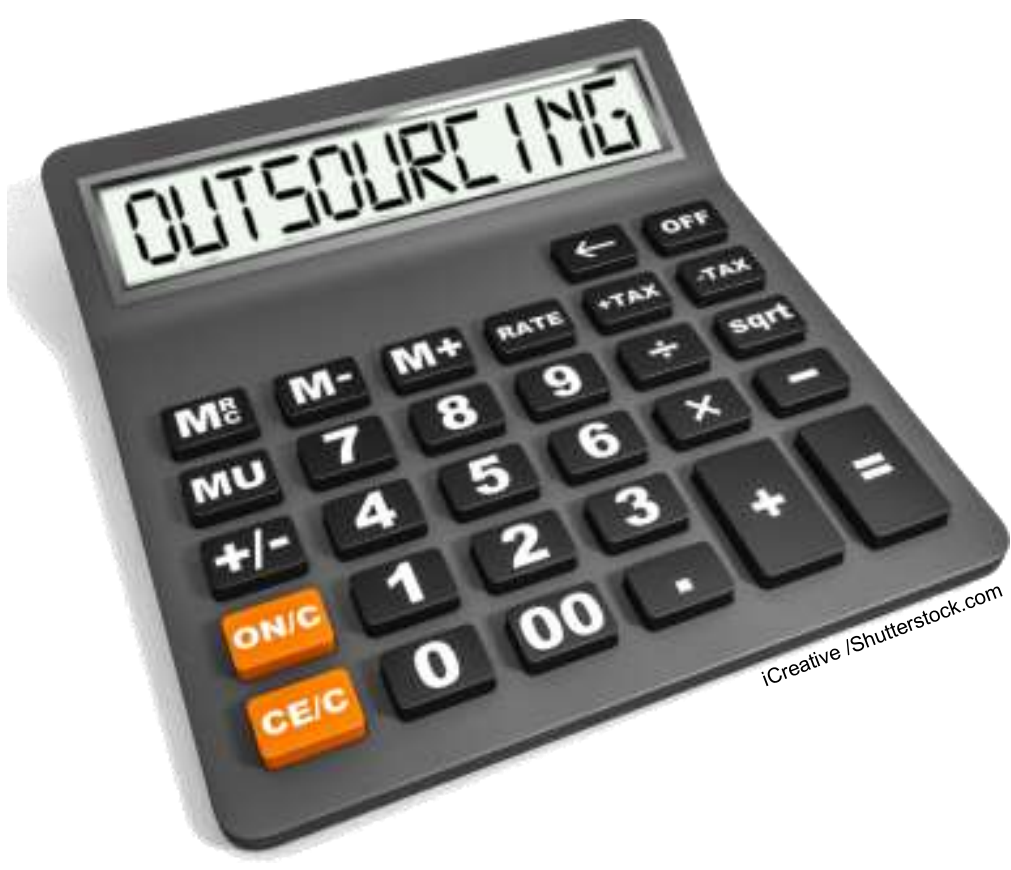

\section{RESUMEN}

En esta investigación empírica se analiza el grado de uso del outsourcing de contabilidad, como herramienta que agrega competitividad y valor a las MiPyMEs de la ciudad de Panamá, su grado de conocimiento de las alternativas que posee esta herramienta, así como su grado de conocimiento e interés por la adopción de las nuevas Normas Internacionales de Información Financiera (NIIF) para PYME. La metodología utilizada, consistió en una encuesta que se realizó con la ayuda de un cuestionario compuesto por diez preguntas cerradas para hacer la recogida de la información más eficiente, aplicado cara a cara al contador de una muestra de 144 MiPyMEs, para evitar errores en la captura de la información. Los datos fueron analizados con la ayuda del estadístico SPSS. Con los resultados obtenidos, se puede afirmar que la mayoría de las MiPyMEs llevan más de 15 años de estar utilizando el outsourcing de contabilidad para la preparación de reportes a terceros, la nómina y el pago de impuestos y están convencidas de que ha mejorado su gestión administrativa, el cumplimiento con los diferentes entes reguladores de las empresas panameñas y en la obtención de financiamiento bancario; además, sus contadores internos se encuentran en una actualización continuada con relación a las NIIF para PYME para mejorar sus conocimientos e interesados en su inmediata implantación.

\section{PALABRAS CLAVES}

Oursourcing de Contabilidad; MiPyME; valor; competitividad; NIIF para PYME.

\section{ABSTRACT}


This empirical research analyzes the degree of use of the outsourcing of accounting as a tool that adds value and competitiveness to MSME of the city of Panama, the degree of knowledge of the alternatives that has this tool, as well as their degree of knowledge and interest in the adoption of new international standards of financial information (IFRS) for SMEs. The methodology consisted of a survey that was carried out with the help of a questionnaire consisting of ten closed questions to make the collection more efficient, applied to a sample of 144 MSMEs counter face to face, to avoid mistakes in the information capture. The data were analyzed using the SPSS statistical. With the results, we can say that the majority of MSMEs are more than 15 years of being using accounting outsourcing for the preparation of reports to third parties, the payroll and paying taxes and are convinced that it has improved its administrative management, compliance with various regulatory bodies of the Panamanian companies and obtaining of bank financing; in addition, its internal accountants found in an update continued in relation to the IFRS for SMEs to improve their knowledge and interested in their immediate implementation.

\section{KEY WORDS}

Accounting Outsourcing, MSME, value, competitiveness, IFRS for SMEs

\section{Introducción}

a globalización de los mercados ha sido la causa de que las empresas tomen en cuenta el outsourcing de contabilidad para mantenerse competitivas. Lo que obliga a las empresas a modernizar sus procesos y ser más productivas, para lograr así un buen nivel competitivo dentro del mercado nacional e internacional. En la búsqueda de la optimización de los procesos y la productividad, es requerido que las empresas se dediquen más a cumplir con su razón de ser y busquen ayuda de terceros para suplir las demás necesidades. Las empresas micro, pequeñas y medianas (en adelante MiPyMEs) son entidades diferentes de las grandes empresas (GEs). "No son una réplica "enana" en su etapa infantil..." (Cleri, 2007, p. 37); no obstante, necesitan herramientas de gestión para aumentar su competitividad y valor en este mundo globalizado.

A lo largo de los últimos años de la década de los noventa viene tomado auge, en el mundo de los negocios, la contratación de empresas y despachos de contadores con el objeto de hacerse cargo de funciones y/o áreas de contabilidad cuyo manejo originalmente corresponde a la empresa contratante (McNamus, 2006; Heywood, 2001). A esta modalidad de atender las necesidades internas, ya sean éstas operativas o administrativas, se le denomina outsourcing. Esto ocurre, cuando una organización externaliza un proceso de negocio que no forma parte de las habilidades principales de ésta a un tercero especializado (Schneider, 2004). Por habilidades principales se entiende todas aquellas actividades que forman el negocio central de la empresa y en las que se cuenta con ventajas competitivas con respecto a la competencia.

\section{Revisión bibliográfica}

En su esencia, outsourcing implica la transferencia de titularidad de actividades de una organización (cliente) a una organización externa (proveedor). El tradicional acuerdo de outsourcing involucraba la compra de componentes a un fabricante externo, esto ha ido más allá de esa posición táctica a ser más bien una herramienta de plan estratégico. El outsourcing estratégico está destinado a hacer más que crear el ahorro de costos, tiene por objeto general que el cliente mejore para que pueda lograr a largo plazo sus objetivos estratégicos, centrándose en las actividades centrales para su éxito.

A pesar que el outsourcing en la actualidad recibe una gran cantidad de atención de los medios, no es un fenómeno nuevo. Surgió en la década de los ' 80 s como 
respuesta a prevalecientes presiones económicas, tomando muchas formas a través de muchas industrias. En una encuesta de 1997-1998, dos tercios de los ejecutivos indicaron que habían subcontratado procesos a una empresa y que la penetración de mercado del outsourcing se esperaba en un crecimiento del 6\% en 1995 al $10 \%$ en 2000 . Que el crecimiento previsto se superó, con la demanda de subcontratación

excedida en un valor global de \$ 100 millones en 2006. Está claro que muchas empresas se están beneficiando del uso de contratos de outsourcing (Juras, 2007).

Una de las funciones secundarias empezó que externalizarse luego de la informática, logrando grandes

\begin{tabular}{|c|}
\hline El outsourcing de contabilidad empezó a \\
tomar importancia desde el momento en \\
que los dueños de negocios se encontraron \\
renuentes a seguir con la contratación de \\
personal de contabilidad costoso, las \\
cargas sociales y de seguridad de ese \\
personal, todo el equipo necesario para \\
mantener el departamento de contabilidad \\
en óptimas condiciones, para la obtención \\
de la información necesaria en la toma de \\
decisiones, cambios tecnológicos \\
constantes y la actualización continuada \\
del personal de contabilidad.
\end{tabular}

condiciones, para la obtención de la información necesaria en la toma de decisiones, cambios tecnológicos constantes y la actualización continuada del personal de contabilidad; lo importante, la reducción de costos y la dedicación de los recursos a su razón de ser, el cliente (Laube \& Roberts, 2002; Caughey, 2002; O'Connor, 2003).

La calidad del outsourcing de contabilidad se evidenció en encuestas realizadas, donde se probó que el flujo de la información desde el proveedor del servicio hacia el personal de gestión de la empresa cliente fue excelente $\mathrm{y}$ por el debido cumplimiento de las leyes fiscales $y$ de resultados por parte de las empresas de todo el mundo, es el outsourcing de contabilidad (Heywood, 2001). Es considerada una estrategia de negocios conocida como outsourcing, herramienta que permite aumentar el valor económico de las empresas y su competitividad, proporcionando eficiencia y eficacia en sus operaciones administrativas (Schneider, 2004).

El outsourcing de contabilidad empezó a tomar importancia desde el momento en que los dueños de negocios se encontraron renuentes a seguir con la contratación de personal de contabilidad costoso, las cargas sociales y de seguridad de ese personal, todo el equipo necesario para mantener el departamento de contabilidad en óptimas normas de contabilidad (Duganier, 2005).

El outsourcing de contabilidad se viene ofreciendo en varias alternativas principales: Contabilidad para la preparación de reportes a terceros, nómina, auditoría interna e impuestos; a continuación se plantean las ventajas en el uso de esas alternativas.

El outsourcing de auditoría interna es la evolución de la función de supervisión tradicional que incluye un amplio espectro de actividades que agregan valor a las organizaciones. Además, las presiones económicas han obligado a muchas empresas a considerar esta alternativa (Ahlawat \& Lowe, 2004). Por otro lado, se justifica, ya que las empresas privadas relativamente pequeñas, MiPyMEs, que 
normalmente no pueden justificar el costo de por lo menos un tiempo completo de un auditor interno. Estas empresas podrían ser capaces de justificar que tienen una firma de contabilidad que le presta los servicios de auditoría interna sobre una base a tiempo parcial (Aldhizer III, Cashell \& Martin, 2003). Se aclara que las firmas de contabilidad ni sus clientes MiPyMEs son consideradas por las normas de control de la Ley Sarbanes-Oxley (2002, Sec. 209), creada luego del descalabro financiero ocurrido en los Estados Unidos de Norte América, que prohíbe a las empresas que cotizan en la bolsa de valores dar en outsourcing las funciones de contabilidad y financieras (Abbott, Parker, Peters \& Rama, 2007; Aldhizer III, Cashell \& Martin, 2003). Es importante mencionar las ventajas en la reducción de costos operativos, de desarrollo, ventas $\mathrm{u}$ otros, enfocar los esfuerzos de la entidad en sus competencias vitales, aprovechar las mejores prácticas e innovaciones de los proveedores, incrementar la flexibilidad y escalabilidad de las operaciones (Moreno, 2008).

Las oficinas de procesamiento de nómina tienen acceso a centros de procesamiento de gran alcance que han automatizado casi todo el proceso de nómina. Si para las empresas grandes el proceso de la nómina era un problema, para las MiPyMEs era un problema mayor (Bhansali, 2008; Turek, 2008).

La gestión de nómina es más que emitir cheques para cada período de pago, es llevar los controles de las horas trabajadas, de los días de vacaciones por pagar y pagados, fondos de ahorro o los préstamos por pagar $y$ otros descuentos de los empleados (Orozco, 2009); hay que tomar en cuenta la preparación de los formularios mensuales que se deben presentar a la Caja de Seguro Social, la preparación de formularios de fin de año, que debe presentarse a la Dirección
General de Ingresos (DGI) de Panamá y el manejo de las investigaciones de los empleados. En general, la nómina no es una competencia básica para los propietarios de estas empresas. Mediante el outsourcing de nómina, los propietarios pueden acceder a un equipo de expertos para ayudar con los problemas y responder preguntas sobre la nómina, los impuestos, de recursos humanos, planes de jubilación, los programas de pago de primas de indemnización de los trabajadores y las tarjetas de pago (Alter, 2008), por lo que la ventaja competitiva es dejar de lado la tarea de procesar la nómina recurriendo a un servicio de nómina externo. (Couch, 2007).

Las normas de contabilidad emitidas por la DGI, la presentación anual de las declaraciones de renta, el pago de los impuestos es otra de las funciones que deben verse con mucho cuidado, las leyes impositivas cambian casi todos los años, por lo que se necesita de una preparación en impuestos tan importante que algunas universidades de este país han creado maestrías y diplomados en impuestos. Tanto grandes como pequeñas empresas se ven afectadas a la hora de una auditoría fiscal. Estas empresas son sometidas a fuertes demandas en dinero por malas interpretaciones de las leyes fiscales, en cuanto, a los registros de contabilidad, el aspecto de territorialidad, ingresos gravables $\mathrm{y}$ exentos, gastos deducibles y no deducibles, entre otros. Aunado a los diferentes pagos de impuestos y la forma de llenar los formularios hace que estas empresas vean la ventaja de recurrir al outsourcing de impuestos, para dedicarse a funciones más importantes (Gautreau II, 2005; Davis, 2005).

Entre las ventajas del outsourcing de contabilidad, se encuentran: reducción de costos, flexibilidad, especialización de recursos, mayor control sobre la calidad del 
servicio y concentración en actividades de su núcleo central, como una herramienta de transformación operativa, de manera que tenga un impacto positivo sobre la rentabilidad de la compañía y su participación de mercado (Crespo, 2008; Linder, 2007).

El outsourcing de contabilidad ha ido evolucionando a tal punto, que se viene ofreciendo con el uso de la Internet, pero deben investigarse todos los riesgos inherentes que intervienen (Bhattacharya, Behara \& Gundersen, 2003).

Esto a su vez le permite a las MiPyMEs acceso a capital humano especializado y estimular el crecimiento global, de estas empresas, asegurando la presencia en economías en crecimiento (Moreno, 2008).

\section{Materiales y métodos}

En esta investigación se tiene como objetivo el estudio del grado de uso del outsourcing de contabilidad por las MiPyMEs de la ciudad de Panamá, qué efecto causa a estas empresas en cuando al mejoramiento de su gestión administrativa y conocer su grado de conocimiento e interés por adoptar la nueva Normativa Internacional, NIIF para PYME. Se presenta un análisis del comportamiento de las variables con los resultados obtenidos.

La presente investigación se circunscribe a las 302 MiPyMEs que se encuentran en una base de datos de investigaciones realizadas en Panamá. De este total, se extrajo una muestra conformada por 144 empresas tomando en cuenta todos los sectores posibles, donde se incluyeron empresas que utilizan el outsourcing de contabilidad como empresas que no lo utilizan. La muestra se obtuvo con un $95 \%$ de grado de seguridad, $3 \%$ de grado de precisión y un $5 \%$ de tasa de ocurrencia.
Las siguientes hipótesis se han tomado como punto de partida:

En esta primera hipótesis, se quiso contrastar el grado de utilización y aceptación del outsourcing de contabilidad y el nivel y su conocimiento sobre su mejora de la gestión (Smith, Morris \& Ezzamel, 2005; Duganier, 2005; O'Connor, 2003; Laube, 2002).

H1: Las MiPyMEs, al igual que las grandes empresas, hacen uso del outsourcing de contabilidad para realizar sus registros de contabilidad y mejorar su gestión.

Con esta segunda hipótesis, se analizó el nivel de conocimiento y uso de nuevos modelos de outsourcing de contabilidad como posible signo de resisa de la cultura organizacional hacia nuevos modelos de outsourcing de contabilidad en el largo plazo (Abbott, Parker, Peters \& Rama, 2007; Caughey, 2002).

H2: Las MiPyMEs conocen los nuevos tipos de outsourcing de contabilidad y sus aplicaciones, con las ventajas de aumento en competitividad y valor que ello les puede reportar.

En esta tercera hipótesis, se recogió el interés y nivel de implantación de la nueva Normativa Internacional, NIIF para las PYME (IASB, 2009).

H3: Las MiPyMEs, que deberán adoptar la nueva Normativa Internacional, NIIF para PYME, están interesadas en ampliar conocimientos sobre ella para su implementación.

Se utilizó un cuestionario de medición, que consta de la solicitud inicial de los datos generales de la empresa encuestada y 10 preguntas cerradas, dirigidas al contador 
interno de la empresa, en su defecto al encargado de los registros de contabilidad. Para el desarrollo de las preguntas, se tomó en cuenta el contenido de las tres hipótesis indicadas.

En los análisis estadísticos y en la determinación de la confiabilidad del instrumento y otros análisis necesarios para esta investigación se utilizó paquete estadístico SPSS ® versión 19 (IBM, 2010).

Tan pronto como se diseñó la encuesta de medición, se procedió a la selección de las empresas que serían incluidas de acuerdo con la muestra que se indicó anteriormente.

Para evitar errores por falta de respuestas o cuando las personas sólo contestan parte de las preguntas (Bernal, 2006), se procedió a la aplicación directapersonal del cuestionario de medición (cara a cara), con la ayuda de cinco personas debidamente preparadas para este trabajo, hasta terminar con las 144 empresas seleccionadas.

Las respuestas logradas fueron de 144 empresas, lo que representa el $100 \%$ de respuestas válidas, procediéndose a vaciar los datos en el editor de datos del SPSS.

\section{Resultados y discusión}

De las 144 MiPyMEs encuestadas, el $67 \%$ admiten utilizar el outsourcing de contabilidad, mientras que el $33 \%$ no lo utilizan.

Para conocer la antigüedad en el uso del outsourcing de contabilidad para la preparación de reportes a terceros, de las 82 MiPyMEs encuestadas que contestaron que Sí utilizaban el outsourcing de contabilidad, $32 \%$ de las empresas cuentan con diez años, $39 \%$ de las empresas entre once y quince años y $2 \%$ de las empresas más de quince años, por lo que se considera que sí se está utilizando este servicio.

El outsourcing de contabilidad está compuesto por varias alternativas, entre ellas se incluyeron en la encuesta, las siguientes: Contabilidad, Contabilidad y Nómina, Nómina, Auditoría interna e Impuestos. De las 82 MiPyMEs encuestadas que contestaron que Sí utilizaban el outsourcing de contabilidad, $65 \%$ mencionan que están utilizando el outsourcing de contabilidad para la preparación de reportes a terceros, $30 \%$ están utilizando contabilidad para la preparación de reportes a terceros, el de nómina y pago de impuestos, 3 utilizan el de impuestos, 0 el outsourcing de auditoría interna, 2 no contestaron.

En relación con el outsourcing de auditoría interna, que fue la opción no utilizada, ocurre así porque existe el riesgo de perder objetividad e independencia por parte del proveedor del servicio (Abbott, Parker, Peters \& Rama, 2007; Ahlawat \& Lowe 2004; Lowe, Geiger \& Pany, 1999).

Por lo que se concluye que estas empresas, en su mayoría, sí están utilizando el outsourcing de contabilidad (H1).

Es importante conocer si el outsourcing de contabilidad para la preparación de reportes a terceros tiene aplicación útil para las MiPyMEs, de las 82 MiPyMEs encuestadas que contestaron que Sí lo utilizaban: el $71 \%$ de las empresas manifestaron que les permite: mejoras en la administración de la empresa, cumplir con eficiencia y eficacia con las instituciones reguladoras estatales, gestionar directamente sus financiamientos con los bancos, lo que representa un valor agregado; mientras que el $26 \%$ contestaron que no le ayuda y el $3 \%$ no contestaron. 
Se puede afirmar que las MiPyMEs de Panamá conocen el outsourcing de contabilidad y sus aplicaciones (H2).

Se presenta hace poco la necesidad urgente de adoptar las NIIF para PYME, a fin de unificar criterios a nivel global, por lo que se hace necesario el conocimiento que tienen las 144 MiPyMEs encuestadas, el resultado fue sorprendente, $87 \%$ de los contadores internos de estas empresas conocen esta normativa, están interesados en utilizar esta opción, y tienen previsto adoptarlas para el manejo de su contabilidad.

Se puede afirmar, que los contadores de las MiPyMEs de Panamá conocen las normas de contabilidad internacional NIIF para PYME, que fueron publicadas en la sitio Web del IASB en el año 2009, en una versión en el idioma español (IASB, 2009) y que cuentan con la necesidad de conocer lo nuevo que se debe hacer para implementarlas adecuadamente (H3), lo que sería una buena estrategia que se llevará a cabo en forma eficiente (Brache \& BodleyScott, 2006).

En el análisis de correlaciones de Pearson y de Spearman, se pudo confirmar, una vez más, las relaciones entre las variables indicadas en los puntos anteriores, que en algunos casos es significativa al 95\%, es perfecta al $99 \%$ y es lineal al $95 \%$.

Con este análisis se prueban como ciertas las tres hipótesis planteadas que se incluyen en esta investigación.

\section{Conclusiones}

Luego de la realización de esta investigación, se puede concluir que las MiPyMEs de Panamá vienen utilizando el oursourcing de contabilidad en la alternativa de contabilidad para la preparación de reportes a terceros, hace más de quince años.
Las MiPyMEs conocen el oursourcing de contabilidad y sus aplicaciones, que les ayudan a cumplir con los reportes que son exigidos por las instituciones reguladoras de estas empresas, que cuentan con poca estructura administrativa, con poco personal capacitado, colocándolas a la par de las grandes empresas.

Los contadores internos de las MiPyMEs conocen la nueva normativa de contabilidad NIIF para PYME, están interesados en la adopción y se sienten deseosos de su pronta implementación.

En relación con la conclusión anterior, pareciera lógico que la opinión de todos los contadores es esa; por lo que es necesario aclarar que en Panamá existen grupos de contadores que no están de acuerdo con la adopción de las NIIF ni de las NIA y han interpuesto demandas ante la Corte Suprema de Justicia para su rechazo, aunque dichas demandas ya hayan sido rechazadas por la Corte.

\section{Bibliografía}

ABBOTT, L. J.; PARKER, S.; PETERS, G. F. \& RAMA, D. V. (2007) "Corporate Governance, Audit Quality, and the Sarbanes-Oxley Act: Evidence from Internal Audit Outsourcing". Accounting Review, julio, 82(4), 803 - 835

AHLAWAT, S. \& LOWE, D. J. (2004) “An Examination of Internal Auditor Objectivity: InHouse versus Outsourcing". Accounting Review, Auditing, Sep. 2004, 23(2), 149-160

ALDHIZER III, G. R.; CASHELL, J. D. \& Martin, D. R. (2003) "Internal Audit Outsourcing". CPA Journal, Aug. 2003, Vol. 73 Issue 8, p. 38-42, 5p, 1 Color Photograph

ALTER, M. (2008) "Ever-Changing Landscape of Payroll Options". Accounting Today, Nov2008 Payroll Supplement, 22, 21-21 
BHANSALI, C. (2008) "How Profitable is Payroll Processing?" Accounting Today, Nov. 2008 Payroll Supplement, 22, 8-8

BERNAL TORRES, C. A. (2006) Metodología de la investigación. México: Prentice Hall, 2a . Ed., 286 pp. ISBN 970-26-0645-4

BHATTACHARYA, S.; BEHARA, R. \& GUNDERSEN, D. (2003) "The business risk perspective on information systems outsourcing". International Journal of Accounting, marzo, 4(1), 19

BRACE, A. P. \& BODLEY-SCOTT, S. (2006) Implementación: Cómo transformar las iniciativas estratégicas en resultados arrasadores. México: McGraw Hill, 195 p.

CAUGHEY, Matt (2002) "Outsourcing Your Procurement Services”. International Journal of Accounting. Chartered Accountants Journal, Aug 2002, 81(7), 20-22

CLERI, C. (2007) El libro de las PyMEs. Argentina: Ediciones Granica, S. A., 443 pp. ISBN 950641-500-6

COUCH, R. L. (2007) "Who wants to do payroll"? Financial Executive, Jan./Feb. 2007, 7(1), 10-10

CRESPO, A. (2008) “Gobierno y gestión”. España: Revista de la Asociación Española de Empresas de Consultoría, 12/09/2008.

DAVIS, R. A. (2005) “Outsourcing Sparks Returns". Accounting Technology, Jan/Feb2005, 21(1), $37-43$

DUGANIER, Barbara J. (2005) "Finance and Accounting Outsourcing Can Improve Control". International Journal of Accounting, Bank Accounting \& Finance (08943958), Jun./Jul. 2005, 18(4), 39-42

GAUTREAU II, J. C. (2005) "Outsourcing will improve quality”. Accounting Today, 8/8/2005, Vol. 19 Issue 14, p. 6-23, 2 p.

HEYWOOD, J. B. (2002) El dilema del outsourcing, la búsqueda de la competitividad. España: Prentice Hall, 202 pp. ISBN 0-273-65617-1

IBM (2010) Guía breve de IBM SPSS Statistics 19. SPSS Inc. 186 pp.
IASB (2009) Norma Internacional de Información Financiera para Pequeñas y Medianas Entidades (NIIF para las PYMES). Inglaterra: IASBF, 257 pp. ISBN 978-607-7620-32-7

JURAS, Paul (2007) "A risk-based approach to identifying the total costo of outsourcing". Management Accounting Quarterly, 9(1), 43-50

LAUBE, J. \& Roberts, Mike (2002) "When you add up the benefits, outsourcing accounting duties saves dollars and makes sense". International Journal of Accounting, Nation's Restaurant News, 3/18/2002, 36(11), 38

LINDER, J. C. (2007) Outsourcing: una estrategia de cambio. USA: Quality Press Editores, 20 p.

LOWE, D. J.; GEIGER, M. A. \& PANY, K. (1999) "The Effects of Internal Audit Outsourcing on Perceived External Auditor Independence". Auditing, 1999 Supplement, 18(2), 7

McNAMUS, Ken (2006) "Is outsourcing an option for Chartered Accountancy firms?" International Journal of Accounting, Chartered Accountants Journal, Apr. 2006, 85(3), 57-59

MORENO, GIL ABAD (2008) "La auditoría interna como outsourcing". Ponencia en la Conferencia Interamericana de Auditoría y Tecnología, Orlando, Florida 16, 17 y 18 de marzo de 2008. Recibido del autor.

O'CONNOR, J. (2003) "Trends in Outsourcing Finance and Accounting”. International Journal of Accounting, Bank Accounting \& Finance (08943958), Oct. 2003, 16(6), 50-52

OROZCO C., L. A. (2009) Estudio integral de la nómina. México: Ediciones Fiscales ISEF, S. A., 8a. Ed. 419 pp. ISBN 978-368-7265-38-2

SARBANES, P. \& OXLEY, M. G. Oxley (2002) Sarbanes-Oxley Act of 2002. Pub. L. No. 107204, 116 Stat. 745

SCHNEIDER, B. (2004) Outsourcing, la herramienta de gestión que revoluciona el mundo de los negocios. Colombia: Editorial Norma, 287 pp. ISBN 9972-895-26-2

SMITH, J. A.; MORRIS, J. \& EZZAMEL, M. (2005) "Organisational change, outsourcing and the impact on management accounting". Accounting Review, diciembre, 37(4), 415-441 
TUREK, W. (2008) "Payroll Outsourcing Goes Beyond Payroll". Accounting Today, Nov. 2008 Payroll Supplement, 22, 16-16

\section{Información del autor}

Candidato a Doctor por la Universidad de Panamá, Catedrático de Contabilidad con el uso de la informática en la Universidad de Panamá, en la que imparte docencia desde el año 1982. Es profesor Titular III de tiempo completo del Departamento de Sistemas de Computación y Administración, para estudiantes de contabilidad y de administración de empresas. Contador Público \#1429, registrado ante la Junta Técnica de Contabilidad, del Ministerio de Comercio e Industrias. Es autor de los libros: Contabilidad General ARPÍA, Contabilidad Agrícolo-Ganadera, Contabilidad Bancaria, Consultas a la Dirección General de Ingresos. Es Magíster en Administración de Empresas. Es Socio Vitalicio y ha formado parte de diversas comisiones del Colegio de Contadores Públicos Autorizados de Panamá, siendo Presidente de la Comisión de las NIIFs, es miembro de la Junta Directiva del Colegio de Contadores Públicos Autorizados de Panamá. Es miembro del The Institute of Internal Auditors (IIA). Es socio fundador de la firma de contadores: Changmarín \& Asociados desde el año 1967. Es Socio Adherente de la AIC. Es Vicepresidente de la Comisión de Ética y Ejercicio Profesional de la AIC. Ha participado en congresos nacionales e internacionales. Presentó un Trabajo Nacional en la XXIX Conferencia Interamericana de Contabilidad 2011, celebrada en Puerto Rico, el que fue aprobado para su exposición y publicación. La AIC le otorgó el Diploma de honor al mérito profesional en el año 2012. eMail: capsa@cableonda.net 